\title{
Impact of Users' Experience with a Web-Based Reporting Portal on Timeliness and Quality of Reporting
}

\author{
Lixuan Wang*, Nina Rothschild and David Lee
}

New York City Department of Health and Mental Hygiene, Queens, NY, USA

\section{Objective}

As part of New York City Department of Health and Mental Hygiene's (NYC DOHMH) efforts to improve provider reporting, the Division of Disease Control surveyed and conducted focus groups with users of a web-based reporting portal called Reporting Central (RC) to learn about their experience with submitting provider reports through $\mathrm{RC}$ and the impact of their experience on data submission.

\section{Introduction}

The New York City Department of Health and Mental Hygiene's (NYC DOHMH) Division of Disease Control (DDC) conducts surveillance of more than 90 specific diseases and conditions and relies on both provider reports and electronic laboratory reports for data. While laboratory reports provide vital laboratory data and represent the majority of the surveillance data that DOHMH receives, they are not always timely or sufficient to confirm a case. Provider reports, in contrast, contain data often not available in laboratory reports and can be more prompt than laboratory reports. Health care providers submit provider reports through multiple channels, including through mailing or faxing paper forms, phone calls, and Reporting Central (RC). In 2016, providers used $\mathrm{RC}$ to submit $\sim 51,000$ provider reports.

\section{Methods}

In June 2017, we used phone calls and messages posted on $\mathrm{RC}$ 's homepage to recruit a convenience sample of $\sim 50 \mathrm{RC}$ users who agreed to participate in a survey and focus group. Participants were assigned to one of five focus groups based on the type and size of the facility where they worked at the time of recruitment: large outpatient facilities $(n=2)$, small outpatient facilities $(n=1)$ and inpatient facilities $(n=2)$. Participants were asked to complete a 14-question paper survey before the focus group. Using a discussion guide informed by DOHMH surveillance subject matter experts, a moderator facilitated discussions on 1) facilitators of/barriers to using $\mathrm{RC}$ and 2) recommendations for improving RC. Each focus group lasted $\sim 90$ minutes. The discussions were audio- and video-recorded and transcribed. Survey results were descriptively summarized with Excel. Focus group data were thematically analyzed with Nvivo by two coders.

\section{Results}

Forty-seven participants responded to the survey, and 45 individuals from a total of 37 health care facilities joined the focus groups.

About $70 \%$ of survey participants rated the difficulty level of $\mathrm{RC}$ as 3 or less on a 1-7 scale (with 7 being the most difficult), and $30 \%$ of participants rated the difficulty level as 1 . Participants from inpatient facilities rated $\mathrm{RC}$ as more difficult to use (mean rating=3.2) compared with participants from large outpatient facilities (mean rating=2.5) and participants from small outpatient facilities (mean rating=1.8). Survey respondents from inpatient facilities reported taking 3-30 minutes (mean=11.4) to submit one report using $\mathrm{RC}$, compared with 3-15 minutes (mean $=7.8$ ) for survey respondents from large outpatient facilities and 3-10 minutes (mean=4.4) for survey respondents from small outpatient facilities.
In subsequent focus group discussions, the majority of participants said that RC is intuitive, the section flow is easy to follow, and training new users requires little effort. Participants with experience using paper forms stated that reporting through $\mathrm{RC}$ is quicker and easier than reporting via paper forms.

Two themes emerged from the analysis of focus group data, revealing the impact of participants' experiences with submitting provider reports on timeliness and quality of data reporting.

Timeliness of Data Submission

Participants noted that flawed functionalities (e.g., lack of auto-save functionality and insufficient time before automatically getting logged out of RC) lead to delayed data submission. Participants from inpatient facilities demonstrated more familiarity with time requirements for reporting and acknowledged the priority of submitting reports in their daily work routine. Participants from outpatient facilities, by contrast, did not acknowledge this priority when describing their reporting workflow and showed less understanding of the importance of timely reporting. Participants from small outpatient facilities questioned the necessity of requiring providers to report because DOHMH is also receiving data from laboratories.

Quality of Data Submission

Participants noted the complexity of selecting the correct data from a long drop-down menu that populates from previous saved entries as a possible contributor to erroneous data entry. Lack of access to some required data and the omission of fields in RC for capturing some relevant data such as patient's gender, housing status, etc. also compromise quality.

\section{Conclusions}

The majority of participants stated that RC is intuitive and easy to use compared to paper forms. This finding encourages us to promote $\mathrm{RC}$ adoption among health care providers who currently use paper reports or do not report.

Focus group participants' proposed enhancements to RC to facilitate timeliness, and quality of data submission include 1) enabling auto-save or save function to reduce data loss in case of crash and automatic log-out, and 2) increasing the amount of time for completing the report, including the amount of time during which the computer is inactive, before automatic log-out. This second enhancement might be particularly helpful for inpatient facilities that frequently report complex cases.

The findings also suggest the potential value of educating health care providers, especially at small outpatient clinics, about the importance and necessity of timely data submission.

\section{Keywords}

Provider Reporting; Web-based Reporting Portal; User's Experience; Timeliness and Quality of Disease Reporting

\section{*Lixuan Wang}

E-mail: Iwang4@ health.nyc.gov 